\title{
RECENT MEASUREMENTS \\ OF THE LOWER \\ THERMOSPHERE STRUCTURE
}

\section{GPO PRICE \$}

CFSTI PRICE(S) \$

\section{SEPTEMBER 1967}

Hard copy $(\mathrm{HC}) \frac{5.00}{65}$

\# 653 July 65

NASA

GODDARD SPACE FLIGHT CENTER GREENBELT, MARYLAND
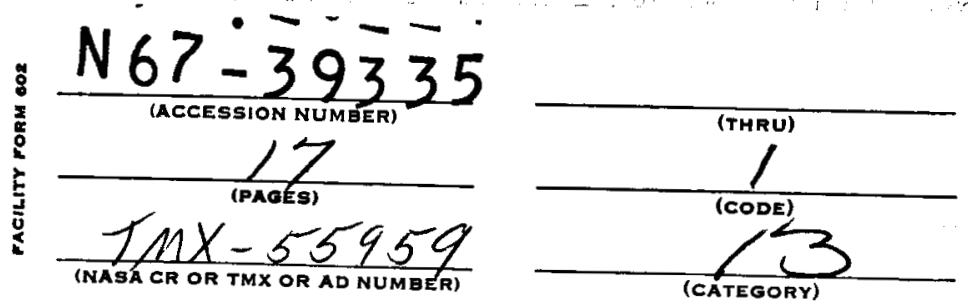
RECENT MEASUREMENTS OF THE LOWER THERMOSPHERE STRUCTURE

N. W. Spencer

G. R. Carignan*

D. R. Taeusch*

September 1967

GODDARD SPACE FLIGHT CENTER

Greenbelt, Maryland

* The University of Michigan, Ann Arbor, Michigan 


\title{
PRECEDING PAGE ELANK NOT FILMED.
}

\begin{abstract}
Measuring of the concentration of neutral particles in the thermosphere poses difficult problems that have long been the subject of considerable experimental development. Recent technological advances now permit new measurements, and the new data show high variability of the temperature and concentration of the constituents in response to variations in the energy absorbed. For example, at times of very low solar activity, densities deduced from satellite drag show little diurnal variation at 200 kilometers, but substantial variation as the altitude increases, reflecting approximately a factor of three (maximum to minimum) at 300 kilometers. In contrast, densities and temperatures measured in the lower thermosphere by mass spectrometer techniques at times of low solar activity show significant variation. At 200 kilometers, where the concentrations of molecular nitrogen and atomic oxygen are approximately equal, the ratio of maximum daytime to minimum nighttime is observed to be approximately 1.8. Recent data, however, do not entirely support these conclusions, showing less variability at the 200 kilometer level with increasing solar activity.
\end{abstract}


PRECEDING PAGE BLANK NOT FILMED.

CONTENTS

$\underline{\text { Page }}$

INTRODUCTION ......................... 1

THERMOSPHERE PROBE EXPERIMENT . . . . . . . . 2

Typical Temperature Results from Thermosphere Probe . . . . 3 SIMULTANEOUS ROCKET SATELLITE EXPERIMENT $\ldots . . . .77$ COMPARISON WITH EXPLORER $32 \ldots \ldots \ldots$ FUTURE PROGRAM .................. 12 REFERENCES ................... 13 


\section{RECENT MEASUREMENTS OF THE \\ LOWER THERMOSPHERE STRUCTURE}

\section{INTRODUCTION}

Although the title of this paper refers to recent measurements of lower thermosphere structure, I would really like to speak on matters which pertain directly to the title of the session, "Measuring Techniques, Accuracies, and Basic Results." Further, I would like to speak about several topics, the first involving generalities relative to measurements and the state of measurements in the lower thermosphere. I will then discuss our Thermosphere Probe experiments, including some results of a number of them. Next I will show some first results of an experiment conducted with rockets during the passage of the Explorer 32 satellite. Finally, I would like to say a few words regarding the future course of our measurement program as it relates to the lower thermosphere.

I think it is important to recognize that we are either passing, or have already passed, what I consider to be an important milestone in our studies of the thermosphere. We have been conducting measurements for a number of years and have now vastly increased our knowledge of at least the gross aspects of the thermosphere. With this knowledge, more precise measurements are needed which will serve the continuing purposes of (a) defining the atmosphere, and (b) testing current theories regarding the physics of the region. Thus, I feel that the time has essentially passed when, for example, the measurement of single profiles of temperature, electron density, etc., serves any particularly useful purpose. We have learned, as has been noted extensively during this meeting, that there are large and significant variations of properties of the high atmosphere that are determined to a great extent by variations of properties of the lower thermosphere. Thus, with the exception of the determination of a very few structural parameters, such as the atomic oxygen distribution, experimentation should now be directed toward conducting combined experiments on a single rocket, or experiments with a multiple rocket approach, that are directed toward specific tests of theory. Only in this way can we learn more about the many factors that influence the physics of the region, and thus advance our understanding.

The recognition of the great variability of atmosphere parameters brings to mind the controversy regarding the lack of close agreement between atmospheric density data as determined from satellite drag and that determined by mass spectrometers and pressure gauges. It is generally recognized that the drag technique is a valuable one, providing a large volume of data from which the 
gross characteristics of the neutral atmosphere can be determined. But it must be remembered that the results obtained from the drag technique represent averages over at least several orbits and thus should not be expected to yield detailed information. In contrast, the gauge techniques provide comparatively few data, making the determination of gross aspects more difficult. However, the gauge results-being derived essentially from measurements made at a point-thus are not averages; therefore they make possible the determination of fine details of the atmosphere and permit studies of the physics of the numerous regions of interest about the earth. Explorer 32 has provided a reasonably large volume of direct measurement data, having made measurements during something like 10,000 four-minute sampling periods distributed among 20 to 25 predetermined locations about the earth. These data will permit, within their limitations, some illustration of global and gross properties; but more importantly, they will provide extensive detailed data of local significance.

Thus, it seems to me that one should abandon concern about the apparent difference between the drag and gauge data and recognize that these techniques provide two different results, both of which are valuable, and both of which will contribute to our understanding of the atmosphere. It seems likely that the difference in the two results will disappear in due course as our understanding improves. Two interesting papers speak to these points; one, by Cook ${ }^{1}$, summarizes the present data from both drag measurements and gauges, concluding that there is a real systematic difference that, to date, is unexplained. A second paper, by $\mathrm{Moe}^{2}$, attempts to ascribe the difference to adsorption and desorption properties of the gauge. I feel that the treatment in that paper is not adequate or correct in explaining the difference, since, for example, it does not consider data to be discussed presently which are in full agreement with other gauge-determined results.

\section{THERMOSPHERE PROBE EXPERIMENT}

I would like now to describe and summarize our Thermosphere Probe experiment and convey to you some of the results obtained, and some of the questions posed. The original objective of the experiment was to study the lower thermosphere and, to this end, we attempted to determine the parameters which could be measured with the greatest confidence, which would permit fruitful studies of the region. Thus, we chose to measure the concentration of molecular nitrogen as the primary experimental objective, as this would permit determination of the neutrai particie temperature. A second objective involved measuring the electron temperature and density by the Langmuir probe approach, which we had developed previously, since knowledge of these parameters along with the molecular nitrogen profiles would permit an assessment of energy transport processes of the region. 
We chose to measure $\mathrm{N}_{2}$ for a number of reasons, including the fact that it is very plentiful. It is also very stable chemically, and thus simplifies the use of a mass spectrometer. In this regard, an important consideration is that the measurement approach can be based in a straightforward manner upon kinetic theory, which permits, in the final analysis, an acceptable experimental error. An additional important factor is that molecular nitrogen mass spectrometers can be calibrated reasonably accurately in the laboratory, a circumstance which does not obtain with, for example, atomic oxygen. The basic chemical stability of the gas permits the use of an orificed chamber, a factor of fundamental importance in establishing the theoretical validity of the measurement.

In conducting the measurements other additional factors, which are illustrated in Figure 1, were considered important. The instruments are housed in a container which is ejected from the rocket carrier when the measurement region is reached. This provision has several desirable features. The moments of inertia of the device, and the manner of ejection, ensure that the probe will tumble end-over-end in a plane, throughout the trajectory. This is a simple motion whose angular description is relatively easy and is redundantly attained through use of an optical aspect sensor. A second direct result of the ejection feature is that comparable data are obtainable during both the up-leg and downleg of the flight without concern for rocket contamination.

This experiment has now been conducted a number of times and is believed to be reasonably fully understood. The errors have been studied at considerable length and include the following. First, and believed to be the most significant source of error, is in the calibration of the omegatron mass spectrometer sensor. The state of the art today permits about 20 to 25 percent absolute accuracy in calibration at the density levels of importance here. Additional errors in the measurement include those due to imprecise knowledge of the trajectory, and the angular motion. These errors, however, contribute only a few percent, and are not really significant. Additional error of the order of 10 percent results from imprecise knowledge of the accommodation effect; that is, how well the gas in the sensor takes on the temperature of the chamber walls. A related error, which is effectively eliminated by correction, relates to how well the chamber pressure follows the theoretical variation indicated by kinetic theory.

\section{Typical Temperature Results from Thermosphere Probe}

A number of Thermosphere Probe experiments have now been conducted over the last several years and in general have been very successful. Of the 12 flights, 11 are considered complete successes. In this paper, results from 8 of these 11 will be shown. It is noteworthy that although most launchings were conducted under "normal" conditions to permit study of the "normal" baseline 


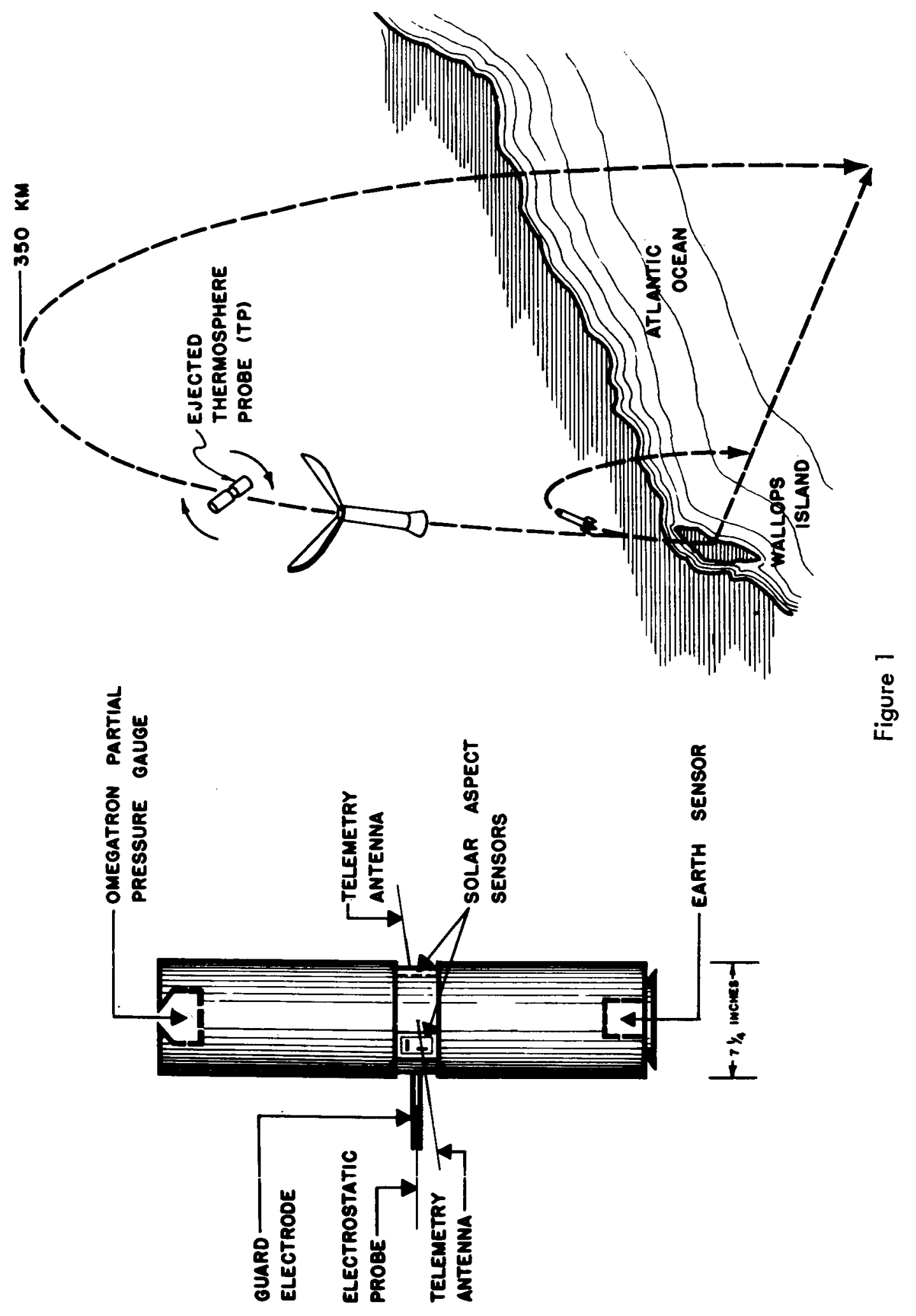


atmosphere, one was conducted during the solar eclipse of July 20, 1963 and produced the very interesting result that the neutral particle temperature decreased rapidly to nearly the nighttime value; this particular result was discussed in an earlier paper. ${ }^{3}$ Table 1 summarizes the successful flights to date.

Table 1

\begin{tabular}{|l|c|c|c|c|c|c|l|}
\hline $\begin{array}{c}\text { Flight } \\
\text { Identification } \\
\text { Number }\end{array}$ & $\begin{array}{c}\text { Density } \\
\text { at 200 km } \\
\text { particles } \\
\mathrm{cm}^{3}\end{array}$ & $\begin{array}{c}\text { Exospheric } \\
\text { Temperature } \\
\left({ }^{\circ} \mathrm{K}\right)\end{array}$ & Date & $\begin{array}{r}\text { Local } \\
\text { Time }\end{array}$ & $F$ & $A_{\mathrm{p}}$ & \multicolumn{1}{|c|}{ Location } \\
\hline 6.06 & $1.5 \times 10^{9}$ & 875 & $11 / 20 / 62$ & 1642 & 89 & 3 & Wallops \\
6.07 & $1.8 \times 10^{9}$ & 940 & $4 / 18 / 63$ & 1602 & 87 & $6-18$ & Wallops \\
6.08 & $2.7 \times 10^{9}$ & 720 & $7 / 20 / 63$ & 1655 & 83 & $4-12$ & Wallops (Eclipse) \\
6.09 & $6.8 \times 10^{8}$ & 730 & $1 / 29 / 64$ & 2209 & 76 & 18 & Wallops \\
6.11 & $1.04 \times 10^{9}$ & 735 & $3 / 20 / 65$ & 0042 & 75 & $4-5$ & Wallops \\
18.01 & $1.69 \times 10^{9}$ & 850 & $3 / 20 / 65$ & 1309 & 75 & 6 & Wallops \\
$18.02>$ Pair & $1.43 \times 10^{9}$ & 654 & $11 / 10 / 65$ & 0100 & 80 & 5 & Churchill \\
18.03 & $8.15 \times 10^{9}$ & 800 & $11 / 09 / 65$ & 1316 & 80 & 6 & Churchill \\
18.05 & $3.9 \times 10^{9}$ & 982 & $8 / 26 / 66$ & 1331 & 130 & 6 & Wallops \\
18.22
\end{tabular}

Figure 2 shows $\mathrm{N}_{2}$ temperature data resulting from six launches during the period of solar minimum. Included for comparison are appropriate model atmosphere temperatures from the Harris and Priester 1964 Model (now CIRA 1965). The conditions pertinent to each flight are shown sequentially at the top of the figure, including the solar flux prevailing at the time of the flight, as well as the geomagnetic index, $A_{p}$. Two day-night pairs are included, reflecting the results of experiments conducted 12 hours apart, and at high and medium latitudes.

A number of points can be made as a result of these experiments and I will note a few here. Of particular interest to this conference is the large variability noted at 200 kilometers altitude and below, in obvious contrast to present models. Note especially the third and fifth curves, labeled W, which reflect a daynight pair of launches conducted at Wallops Island, showing that the gradient of the nighttime profile exceeds that of the daytime profile. Although we believe 


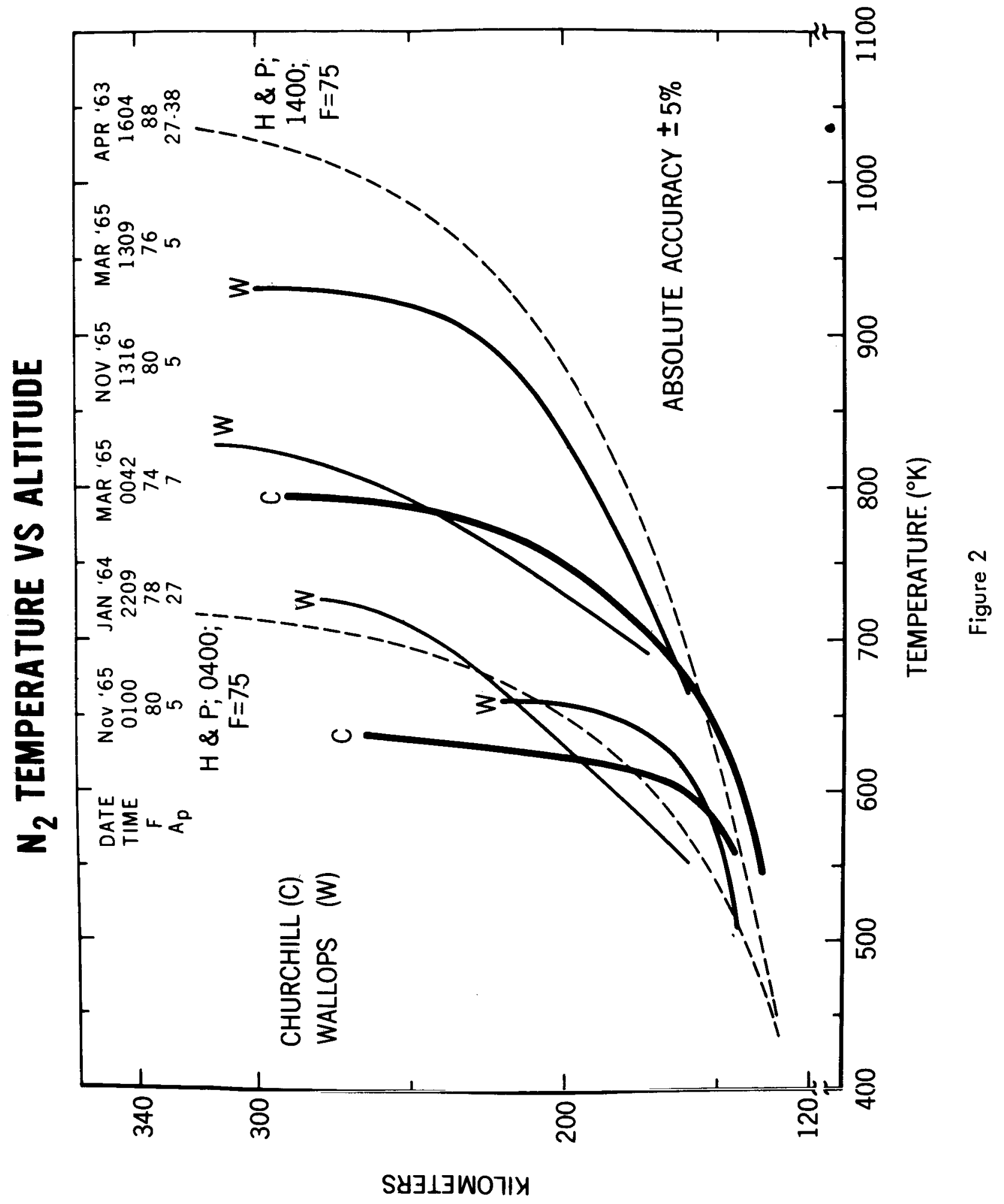


these data to be correct within 5 percent, a clear explanation of this apparent anomaly is not at hand. It is to be noted that there was apparently no significant change from day to night in either the solar flux or the magnetic index. Also notable is number six curve (left to right) because of the apparently high temperature. As noted at the top of the figure, the $A_{p}$ for that flight was about 30 . If the measured exospheric temperature is corrected to the same $A_{p}$ as the other data, the resulting temperature value is about $800^{\circ}$, in agreement with the other measured daytime exospheric temperatures. Therefore these data are consistent and indicate that the CIRA 1965 model daytime temperature data are clearly too high.

In summary, the important point for this conference is that the data strongly suggest a significant variability in the atmosphere in the 110 to 120 kilometer region, implying a variable turbopause level and possibly significant stimulation from lower levels. These data clearly indicate that more extensive and intensive study of the lower regions, including additional measurements, and probably a better understanding of lower thermosphere processes, are needed before the data can be interpreted adequately and fully.

\section{SIMULTANEOUS ROCKET SATELLITE EXPERIMENT}

In a more recent experiment, an attempt has been made to obtain more quantitative results regarding the lower thermosphere and, by providing data at lower and higher altitudes, at the same time permit the obvious correlations. In addition, an objective of this experiment involved conducting rocket measurements simultaneously with similar measurements made by the Explorer 32 satellite at the same geographic location to permit additional correlations.

To implement the overall experiment, six rockets were used, and were launched in a tight, time sequence timed to coincide with satellite passage. The launch date was chosen to permit measurements at approximately mid-afternoon, the time of the daily maximum, and during the early morning hours, approximating the nighttime minimum period. Two Pitot-Static Tube launches (see the paper by Wendell S. Smith at this conference for details of this technique) were selected to provide density and temperature profiles from the ground to approximately 110 kilometers, and three Thermosphere Probe launches were conducted to provide nitrogen density and temperature profiles in the thermosphere. In addition, neutral and ion composition, electron density and temperature were measured by part of the Thermosphere Probe instrumentation. An additional launching was conducted by the Air Force Cambridge Research Laboratory under the direction of Dr. Hinteregger to provide measurements of the solar flux input to the atmosphere.

The satellite (Explorer 32) was operated almost continuously during the portion of the orbit near Wallops Island, thus providing measures of the neutral 
density and the electron temperature almost simultaneously with the rocket measurements. The results from the satellite are not yet available in quantity, but one representative computation resulting from the overall experiment is included in this paper.

Figure 3 shows the nitrogen density profiles resulting from four rocket launches. The results correspond to the daytime maximum and nighttime minimum as noted above. The data below 100 kilometers obtained from the PitotStatic Tube experiment are presented in terms of equivalent $\mathrm{N}_{2}$ density derived from the total density measured. Since mixing is complete for these purposes throughout this region, an $\mathrm{N}_{2}$ profile can easily be derived for comparison with the higher altitude data. Although the scales are considerably compressed to enable drawing the figure, and thus the relatively small variations observed at the lower altitudes are obscured, the diurnal variation of the thermosphere is clearly visible down to the lowest measurement altitude, approximately 130 kilometers. It is to be noted that the dashed curves are pure extrapolations, as neither experiment provided useful data in this region. We feel, however, that the region of extrapolation is so limited that a large error is not likely; thus the profiles are believed to be very representative of the entire region from approximately 10 kilometers to 300 kilometers.

These data are particularly noteworthy (in contrast to previous data) in that they are the first Thermosphere Probe density values which are in substantial agreement with the CIRA 1965 model, for the conditions holding at the time of the measurements. Previous data already published ${ }^{3}$ shows consistently lower density than the model. Since we believe all data to date to be accurate on an absolute basis to better than $\pm 25 \%$, we cannot explain this apparent inconsistency and are inclined to believe that the differences are real and reflect causes of variability of the atmosphere at times of low solar activity which are either unknown or not fully understood. There is a strong suggestion that the differences observed between the directly measured data and those extrapolated from drag measurements are peculiar to the period of solar minimum. However, there are insufficient data on hand to confirm or deny a possible correlation.

It is of interest here to note the agreement between the density at 120 kilometers as obtained from Figure 3 (approximately $3.2 \times 10^{11}$ ) and the value noted by Champion earlier in this meeting $\left(3.14 \times 10^{11}\right)$, that had been selected for the new Supplemental Atmosphere Models.

Figure 4 shows temperature profiles derived from the Figure 3 density profiles. As for the Figure 3 data, these data reflect the results from four rockets, and provide the basis for evaluating the day maximum/night minimum ratio. Unfortunately, however, a solar storm in progress at the time of the 


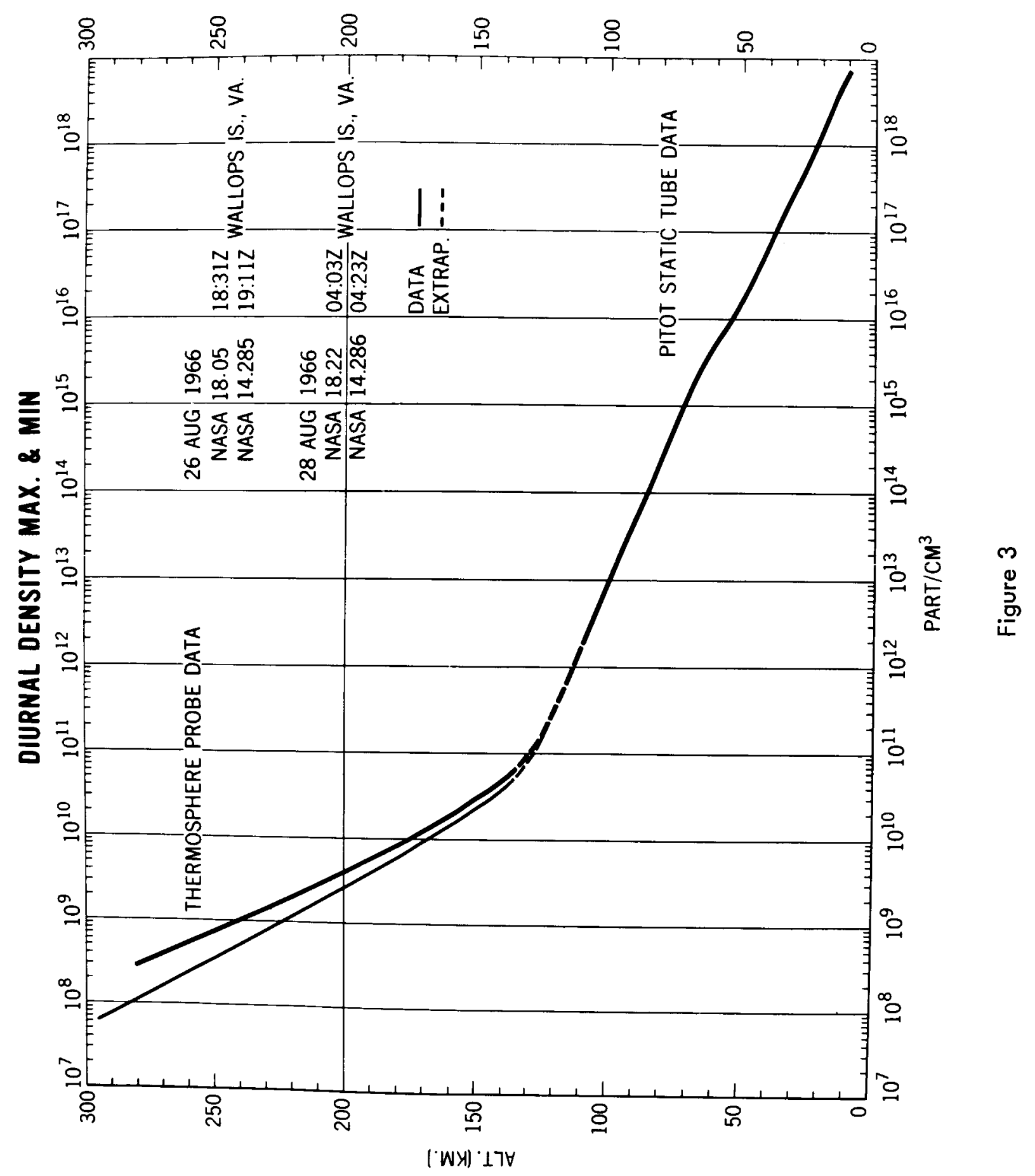




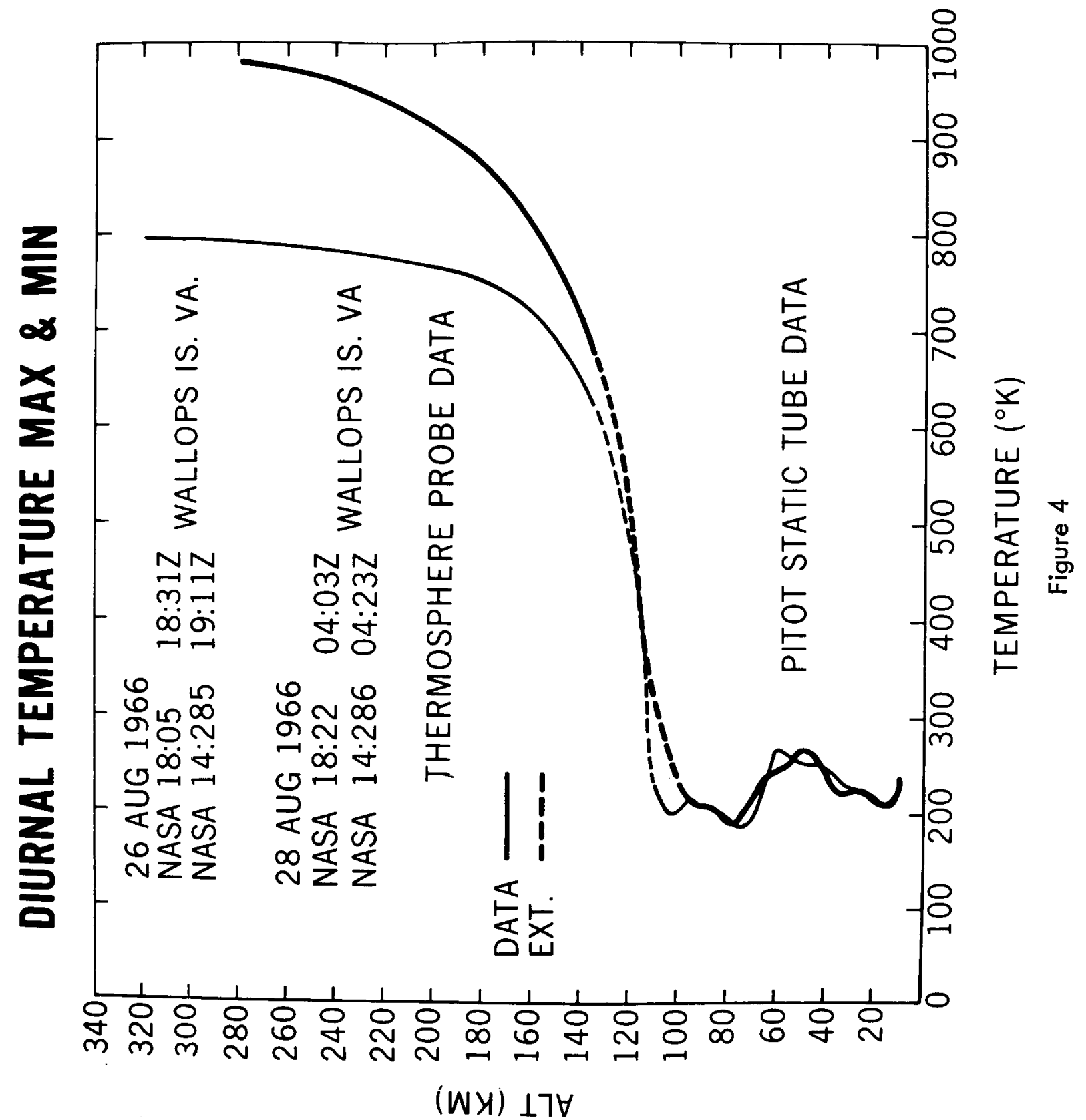


launches resulted in the arrival at the earth of a significant number of protons, which may have affected the nighttime temperature. Our studies have not yet permitted examination of the full details of the storm or the possible effect of the protons, and thus the day-night comparison possibly reflects an abnormal non-quiet nighttime atmosphere.

Again, it is to be noted that these curves (in the 120 kilometer region) result from extrapolating the lower atmosphere data to the higher atmosphere data. The extrapolation of the daytime curve was done independently of the nighttime data, and thus the overlap at 120 kilometers should be considered real. Again, the error is not considered too great in the sense of a smooth atmosphere; thus we believe the extrapolated temperatures in the 120 kilometer region reflect quite well the actual temperatures at the time of the measurements.

A number of interesting points are illustrated by these data. The value indicated for 120 kilometers is about $475^{\circ}$ and is the same for both day and night. This is clearly considerably greater than model boundary values, and supports the concept of significant variability in the lower thermosphere.

Generally, the temperatures in the upper atmosphere appear quite "normal" as compared with earlier Thermosphere Probe data, and do not show the gradient anomaly noted for the earlier data. Two additional points are of interest. The significant difference between the daytime and nighttime temperature in the 100 to 120 kilometer region is quite apparent and is probably fully consistent with the "high" 120 kilometer temperature. No explanation is apparent for the observed variability in the ozone region maximum temperature, other than to note that it is believed that the data properly reflect the true variation of the atmosphere in this region at the time of the measurements.

\section{COMPARISON WTTH EXPLORER 32}

Although the results from these rocket flights and the extensive results from Explorer 32 (not yet available) will, in time, permit extensive and very useful correlations, it is only possible to present one example at this time. Referring to Figure 3, the nitrogen density determined by extrapolating the daytime curve to 316 kilometers is $8.5 \times 10^{7}$ particles per cc (316 kilometers was the altitude of the satellite near the apogee of the rocket trajectory). The corresponding value from the satellite density gauges* is $1.7 \times 10^{-14}$ grams per cc. This value reflects the total amount of gas in the gauge which at this altitude

\footnotetext{
*We are grateful to George P. Newton, GSFC, for this early data point from his Explorer 32 experiment.
} 
is predominantly $\mathrm{N}_{2}$ and $\mathrm{O}$. Using the value of $\mathrm{N}_{2}$ from the Thermosphere Probe and comparing the satellite data (corrected for approximate composition) permits a determination of the $\mathrm{O}$ to $\mathrm{N}_{2}$ ratio at the 316 kilometer level. The value of the ratio thus computed is 5.5 , which we present here as entirely preliminary information. It is interesting to note, however, that it generally supports the ratio obtainable from CIRA 1965 for the $1000^{\circ} \mathrm{K}$ exospheric temperature which existed at the time of the measurement (Thermosphere Probe Data, Figure 4). We present these data as an example of the possibilities that data correlation provides.

\section{FUTURE PROGRAM}

We believe the kind of experiment discussed here, in which simultaneous measurements are made using rockets and satellites, and in which day-night and other comparisons are made, reflect a desirable and increasingly important use of rocket and satellite capability. In this way, existing atmospheric theory can be tested, and probably new theories generated. In this regard, we note particularly that multiple and simultaneous measurements are very important. We plan to continue our thermosphere study program with great emphasis on closely coordinated experiments. Already being studied is another Atmosphere Satellite ${ }^{4}$ which will provide the opportunity to make measurements in the lower thermosphere, at altitudes as low as 120 kilometers, and will thus permit extensive experimentation in that region. Also planned are a number of additional Thermosphere Probe experiments to provide a variety of data under different and selected circumstances. Temperature data such as those shown in Figure 2 clearly indicate the need for carefully planned and extensive studies, for the data strongly indicate not only the variability, but also the existence of apparent anomalies whose explanation will be obtained only through closely coordinated experiments. 


\section{REFERENCES}

1. Cook, G. E., Royal Aircraft Establishment Technical Report No. 66370, "Comparison of Air Densities Obtained from Orbital Decay and Instruments, " November 1966.

2. Moe, Kenneth and Mildred M., "The Effect of Adsorption on Densities Measured by Orbiting Pressure Gauges," Publication No. 576, Institute of Geophysics and Planetary Physics, University of California, Los Angeles, California.

3. Spencer, N. W., Carignan, G. R. and Taeusch, D. R., Extrait des Annales de Geophysique, " $\mathrm{N}_{2}$ Temperature and Density Data for the 150 to $300 \mathrm{Km}$ Region and Their Implications," Tome 22, No. 2, Avril-Juin 1966.

4. Feasibility Study, "Atmosphere Explorers AE-C and D (Low Perigee)," Goddard Space Flight Center, Greenbelt, Maryland, April 1967. 